Kalajdžić, Milan

Momčilović, Nikola

http://dx.doi.org/10.21278/brod71205

ISSN 0007-215X

eISSN $1845-5859$

\title{
A STEP TOWARD THE PRELIMINARY DESIGN OF SEAGOING MULTI-PURPOSE CARGO VESSELS
}

\author{
UDC 629.5.01:629.5.012:629.542.2 \\ Preliminary communication
}

\begin{abstract}
Summary
The preliminary design stage is a starting and vital step in the ship design process which generally involves the adaptation of a prototype selected from an available and trustworthy vessel database. This investigation was prompted by the noticeable lack of systematic literature data on multi-purpose cargo vessels (MPCV), especially in the range of medium size fleet, with respect to gross tonnage. A procedure is presented for optimum MPCV characteristics determination in the preliminary design stage. It is grounded on a statistical analysis of the database gathered upon reliable MPCV built over the past 30 years. Using the proposed set of diagrams and formulas, the designers could determine ship principal dimensions upon required deadweight as a main prerequisite, as well as optimum energy efficiency design index, tank capacities, lightweight, etc.
\end{abstract}

Key words: preliminary design; ship design, multi-purpose vessels; MPCV

\section{$\underline{\text { Nomenclature }}$}

\begin{tabular}{|c|c|c|c|}
\hline$B$ & breadth in $\mathrm{m}$ & $n$ & number of ships \\
\hline$C_{B}$ & block coefficient & $P$ & probability \\
\hline$C C$ & cargo capacity in $\mathrm{m}^{3}$ & $P_{B}$ & $\begin{array}{l}\text { main engine power in } \\
\mathrm{kW}\end{array}$ \\
\hline$C_{F}$ & $\begin{array}{l}\text { conversion factor in } \\
\mathrm{gCO}_{2} / \text { tfuel }\end{array}$ & $P_{M E D A}$ & $\begin{array}{l}\text { main engine alternator } \\
\text { power, } \mathrm{kW}\end{array}$ \\
\hline$D O$ & diesel oil in $\mathrm{m}^{3}$ & $p d f$ & $\begin{array}{l}\text { probability density } \\
\text { function }\end{array}$ \\
\hline$D W T$ & Deadweight in $\mathrm{t}$ & $R^{2}$ & $\begin{array}{l}\text { coefficient of } \\
\text { determination }\end{array}$ \\
\hline$E(X)$ & mean value & $S F C$ & $\begin{array}{l}\text { specific fuel } \\
\text { consumption in } \mathrm{g} / \mathrm{kWh}\end{array}$ \\
\hline EEDI & $\begin{array}{l}\text { energy efficiency design } \\
\text { index in } \mathrm{gCO}_{2} / \mathrm{t} \cdot \mathrm{nm}\end{array}$ & $T$ & draught in $\mathrm{m}$ \\
\hline
\end{tabular}




\begin{tabular}{|c|c|c|c|}
\hline$E E D I_{R e f}$ & $\begin{array}{l}\text { EEDI reference line in } \\
\mathrm{gCO}_{2} / \mathrm{t} \cdot \mathrm{nm}\end{array}$ & $V$ & speed in kn \\
\hline$E E D I_{R e q,-10 \%}$ & $\begin{array}{l}E E D I \text { reference line, } \\
\text { reduced by } 10 \% \text { in } \\
\mathrm{gCO}_{2} / \mathrm{t} \cdot \mathrm{nm}\end{array}$ & $V(X)$ & coefficient of variation \\
\hline$E E D I_{R e q,-15 \%}$ & $\begin{array}{l}E E D I \text { reference line, } \\
\text { reduced by } 15 \% \text { in } \\
\mathrm{gCO}_{2} / \mathrm{t} \cdot \mathrm{nm}\end{array}$ & $W B$ & water ballast in $\mathrm{m}^{3}$ \\
\hline$E E D I_{R e q,-30 \%}$ & $\begin{array}{l}E E D I \text { reference line, } \\
\text { reduced by } 30 \% \text { in } \\
\mathrm{gCO}_{2} / \mathrm{t} \cdot \mathrm{nm}\end{array}$ & $X$ & variable \\
\hline$F_{B}$ & Freeboard in $\mathrm{m}$ & $\beta$ & reliability index \\
\hline$F_{B, I C L L}$ & $\begin{array}{l}\text { freeboard ICLL reference in } \\
\mathrm{m}\end{array}$ & $\Delta$ & Displacement in $\mathrm{t}$ \\
\hline$F_{n}$ & Froude number & $\eta_{D W T}$ & deadweight ratio \\
\hline$G T$ & gross tonnage & $\sigma(X)$ & standard deviation \\
\hline$H$ & height in $\mathrm{m}$ & & \\
\hline $\mathrm{HFO}$ & heavy fuel oil in $\mathrm{m}^{3}$ & $\underline{\text { Abbreviations }}$ & \\
\hline$L$ & $\begin{array}{l}\text { length between } \\
\text { perpendiculars in } \mathrm{m}\end{array}$ & ICLL & $\begin{array}{l}\text { International Convention } \\
\text { on Load Lines }\end{array}$ \\
\hline$L_{O A}$ & length overall in $\mathrm{m}$ & IMO & $\begin{array}{l}\text { International Maritime } \\
\text { Organization }\end{array}$ \\
\hline$L W T$ & Lightweight in $\mathrm{t}$ & MEDA & $\begin{array}{l}\text { Main Engine Driven } \\
\text { Alternator }\end{array}$ \\
\hline$M$ & margin & MPCV & $\begin{array}{l}\text { Multi-Purpose Cargo } \\
\text { Vessels }\end{array}$ \\
\hline
\end{tabular}

\section{Introduction}

The goal of this paper is to provide the practical tools for the preliminary design of multi-purpose cargo vessels (MPCV) in order to determine reliable and optimum ship characteristics. The selection of ship parameters based on a prototype depends on the availability and reliability of a database of similar ships. However, for MPCV, especially those below $200 \mathrm{~m}$ in length or medium sized according to gross tonnage, in between $500-$ $25000 \mathrm{GT}$, there is a visible lack of systematically available resources.

A wide range of MPCV and their design trendlines can be found in the literature, as in [1]. Research presented in [2, 3] also proposes diagrams for the determination of main particulars, but the ships' databases are to some extent outdated. Early design stage objectives and procedures in the case of multi-purpose heavy lift vessels can be found in [4] and [5]. Paper [6] introduced an optimization procedure in case of preliminary design of grain bulk ships. Moreover, [7] examines parametric design objectives and provides a helpful general procedure for the preliminary design of merchant ships. Analogous investigations are conducted for the containerships $[8,9,10]$ and could be carefully applied to MPCV designed with consideration of use in container transport. 
Generally, the literature presents tools for merchant fleet or MPCV at wide range of lengths. Thus, early design tools for medium-sized MPCVs based on contemporary databases need to be updated. Medium fleet belong to a niche market that includes a wider diversification with respect to the cargo, draught, navigation, etc. This paper gathers the reliable resources into the practical use, in order to help the designers to make more thorough decisions.

Optimal ship design involves finding a balance among many parameters [2, 7]. The 'ship design spiral' consists of iterative stages in which the preliminary parameters, represented by the second point of outer loop of Figure 1 [11], are shaped and refined [2, 3, 7, $11]$.

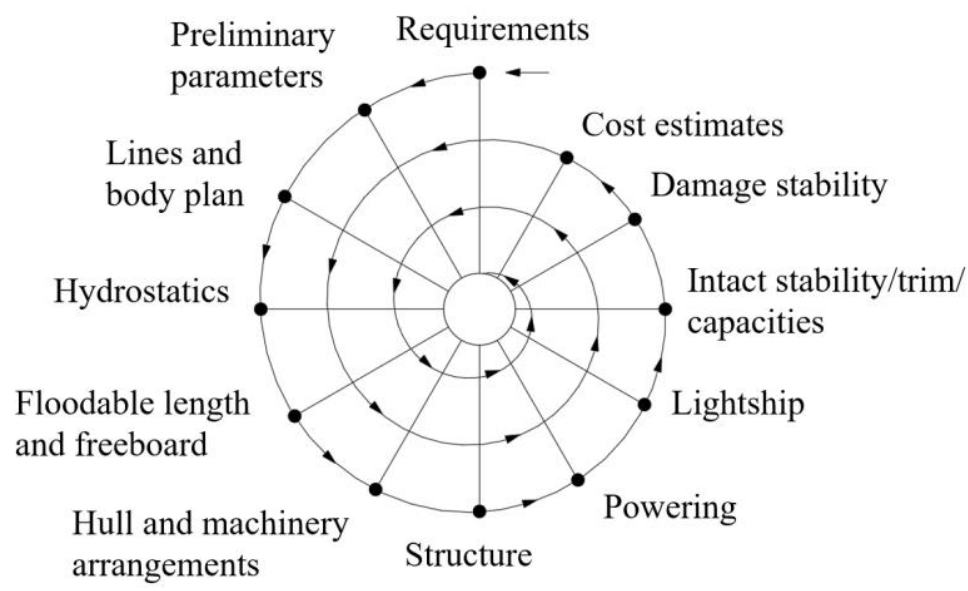

Fig. 1 Ship design spiral

According to [7], the design and engineering can be roughly divided into four phases: concept design, preliminary design, contract design and detailed design. Excluding the general concept design, during which global fleet characteristics are determined, the preliminary design is the first stage of genuine naval architect considerations. Generally, a design procedure starts with a ship design that is based on similar and reliable designs within the existing fleet $[2,3]$. Then, a successful and proven ship from the fleet is chosen to be a prototype and customized to fulfil the requirements in iterative manner, as annunciated in [11]. More thorough state-of-the-art design methodology is presented in [12]. The preliminary stage is important because early estimated main dimensions have an important impact on final ship performance.

Regarding MPCV design objectives, there is still a need in the shipping industry for a flexible solution to optimize transport with regard to cargo - for instance, the accommodation of various goods like containers, pallets, bulk, grains, metal products/scarp, etc. A large number of these ships (e.g., river-sea, sea-river, coastal vessels) usually navigate shorter distances where frequent changes of cargo can be performed readily. Therefore, cargo variations have a significant influence on draught change and hence main dimensions.

Short-sea shipping is a rapidly developing sector, as multi-purpose fleet is recovering from years of stagnation. MPCV make up about $15 \%$ of all medium size ships with respect to GT, up to four years old, less than only the proportion of offshore $-22.5 \%$ and oil/chemical ships - $17.2 \%$, according to [13]. Furthermore, MPCV make up the highest share of all medium fleet, almost $30 \%$. Yet, the MPCV tonnage share in a total fleet is smaller, around $11.3 \%$, due to their limited dimensions [14]. 
MPCV have been evolving over the years since the old ones carried one type of cargo and nowadays, especially large vessels, are intended for different cargoes in a single voyage. On the other hand, smaller MPCV are still designed for one type of cargo. Therefore, according to the main cargo type, MPCV could be categorized as, for instance, more oriented towards container shipping, bulk, short-sea or, for the emerging market as - project carriers, i.e. vessels designed for the single infrastructure project deliveries.

MPCV design requires the consideration of certain structural specifics. For example, the bottom girder and floors are to be fitted for container corners, as most of these ships are suited for container carriage. In addition, the inner bottom is generally strengthened for heavy cargo loading and grab bucket applications. Finally, MPCV are prone to torsion issues due to large hatches along the ship, making their structure very similar to containerships.

\section{MPCV statistics}

A part of the database of reliable MPCV ships is presented in [14] (included vessels built from 1990 to 2013) from data obtained from Significant Ships, a publication [15] that labels the complete particulars of various important and innovative vessel designs. This source is widely perceived as reliable [3]. The database in this paper contains 110 vessels, systematically updating [14] to include more of ship specifics: the power of the main engine driven alternator - MEDA, ship lightweight, water ballast, cargo capacity, fuel oil, etc. Additional newbuilding data were collected from producers' sources, such as [16]. Compared to the database and diagrams presented in [14], the procedure here is based on much more refined data and contemporary MPCV. The research included the vessels built over the past six years and excluded the ones produced before the 1995, making the design trendlines more reliable and offering the insight within the database, through their pdf distributions.

The database is presented in the form of probability density functions, i.e. $p d f$, of ship parameters, where each point accounts for a specific ship. See Figures 2-6 for: $L_{O A}, B, H, T$, $F_{n}, C_{B}, P_{B}, P_{M E D A}, \triangle, D W T, L W T, G T$, water ballast $(W B)$, cargo capacity $(C C)$, diesel oil $(D O)$, heavy fuel oil $(H F O)$. Absent parameters for some ships, such as $C_{B}$, are calculated, since corresponding data are given: $\Delta, L, B, T$. Using $p d f$, one could evaluate the bandwidth of the curves to assess the chosen value of its own ship and moreover, to demonstrate the distribution and the frequency of the particulars in fleet data. The $p d f$ of variable $X$ (which could be any of the presented parameters) is calculated according to the Gaussian distribution, as in equation

$$
p d f(X)=\frac{1}{\sqrt{2 \pi \sigma^{2}(X)}} e^{\frac{-(X-E(X))^{2}}{2 \sigma^{2}(X)}}
$$

Table 1 presents the obtained statistical parameters in the database: the number of available data for all ships $n$, mean value $E$, and standard deviation $\sigma$ of the variable $X$. $X$ stands for ship parameter. Therefore, the presented figures and table give insight into the range of produced and reliable ships, which is important in the preliminary design stage. 


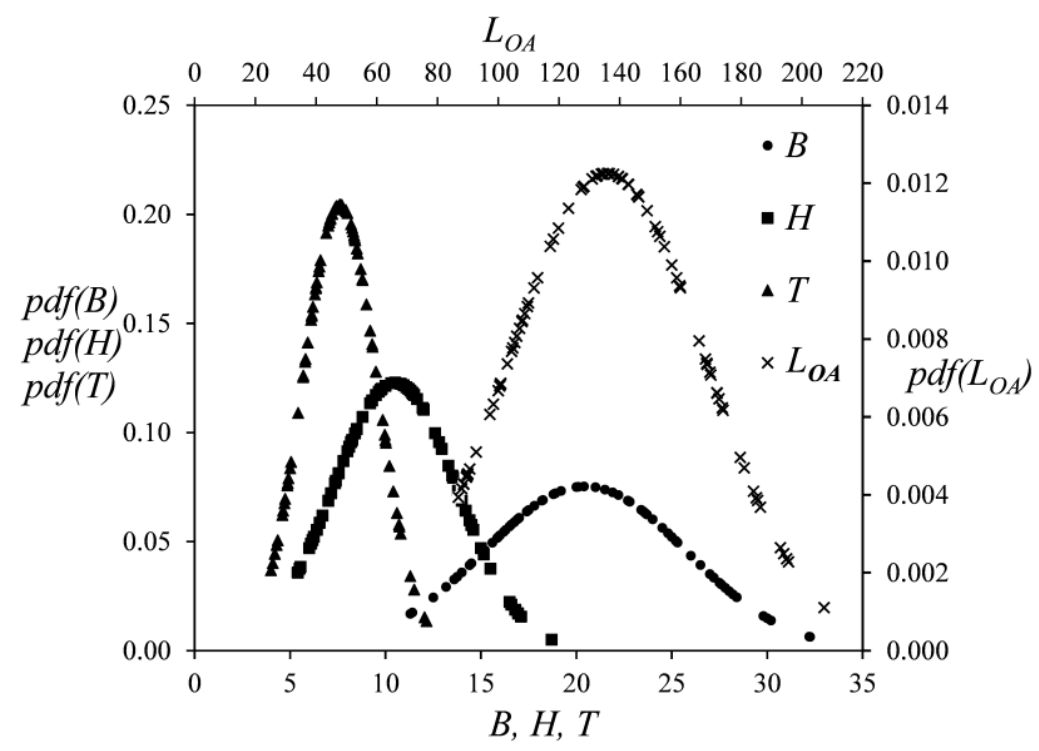

Fig. 2 pdf of $L_{O A}, B, H$ and $T$

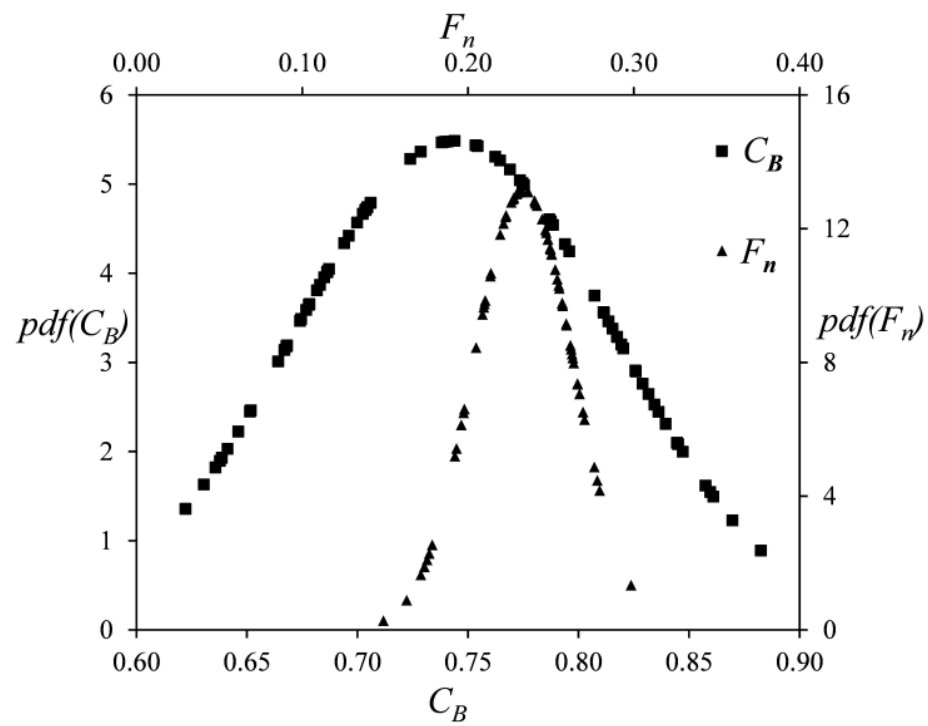

Fig. 3 pdf of $F_{n}$ and $C_{B}$

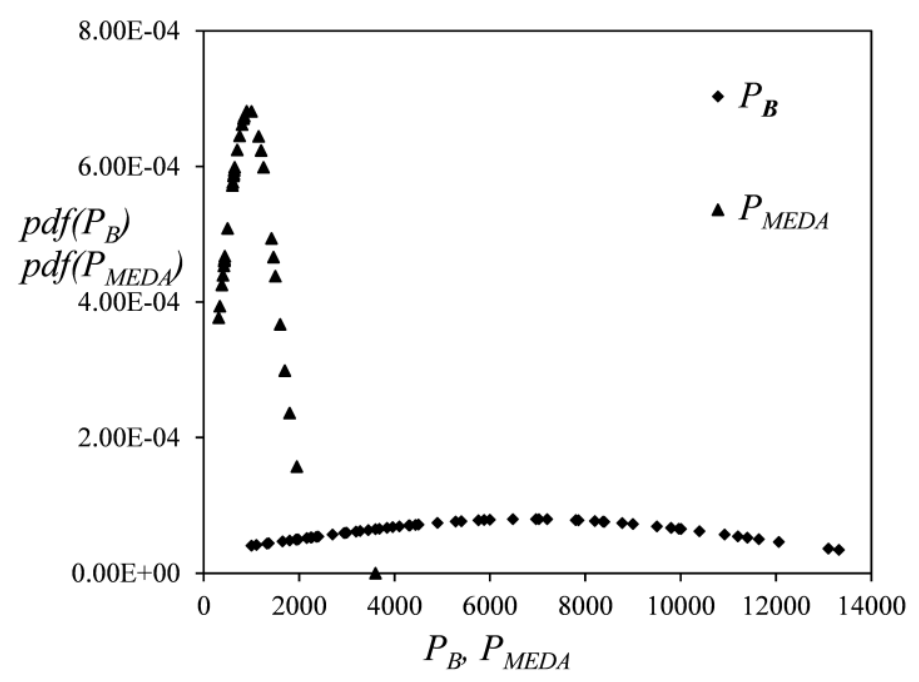

Fig. 4 pdf of $P_{B}$ and $P_{M E D A}$ 


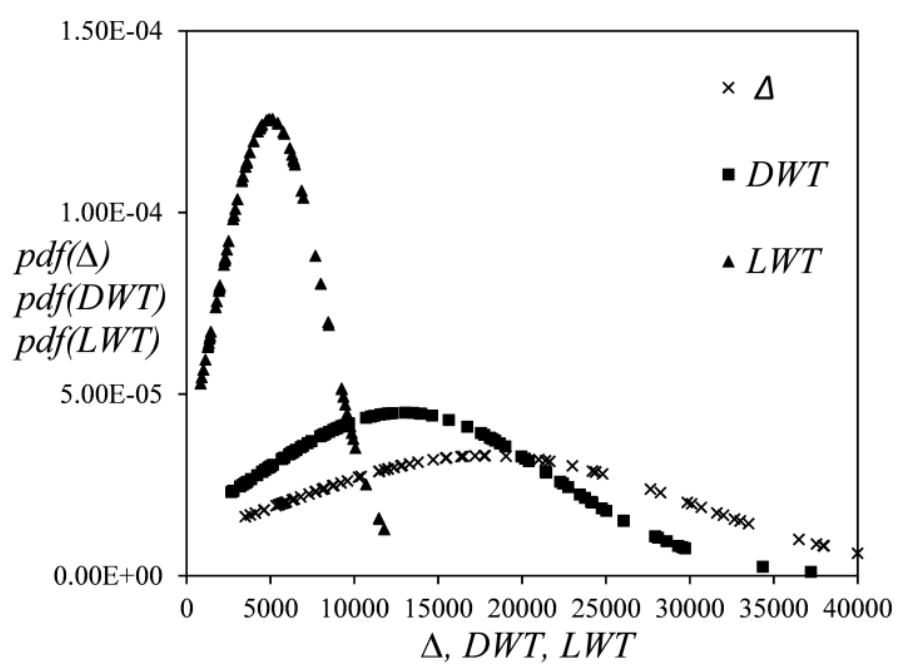

Fig. 5 pdf of $\triangle, D W T$ and $L W T$

$\mathrm{DO}, \mathrm{HFO}$

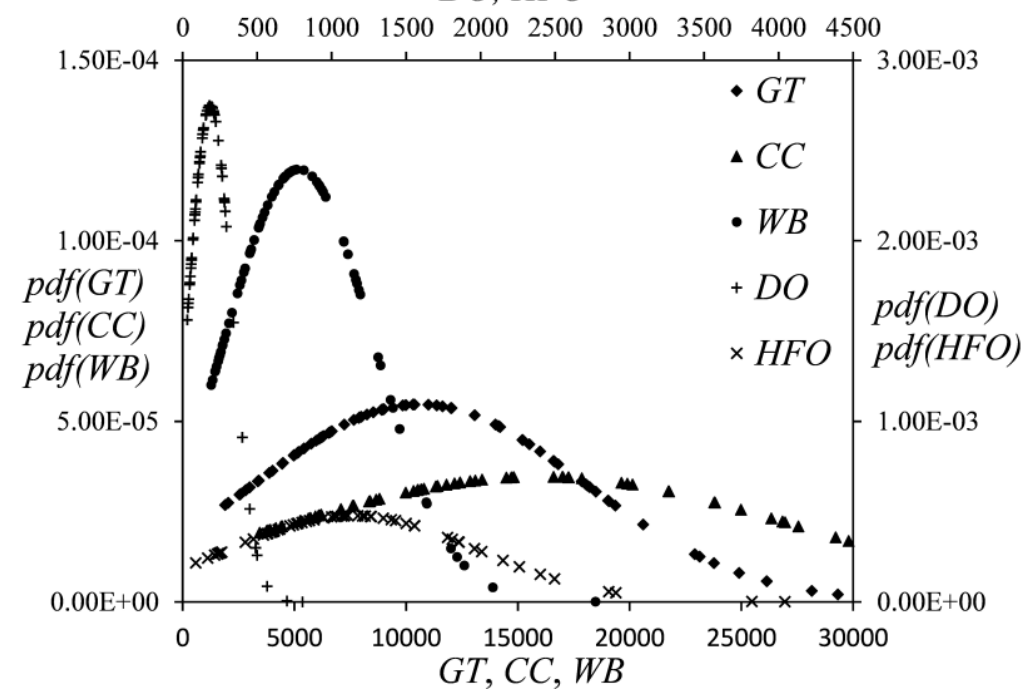

Fig. 6 pdf of $G T, C C, W B, D O$ and $H F O$

Most of the ships have $L_{O A}$ in the 130-170 m range while navigating on $F_{n}=0.20-0.26$. The $p d f$ peak of $C_{B}$ is at 0.74 . Moreover, the range of $P_{B}$ is large, as these ships are diversified considering the frequent change of draught and cargo. $P_{M E D A}$ could be estimated as approximately 7-8 times lesser than $P_{B}$.

Some additional $p d f$ s are calculated, as in Figure 7, based on Figures 2-6, and are to be used in preliminary design. They include: deadweight to displacement ratio - $\eta_{D W T}$; water ballast to displacement ratio $-W B / \triangle$; and $L W T$ to $L B H$ ratio. MPCV have $\eta_{D W T}$ around 0.71 with a slight standard deviation implicating that these ships have relatively similar forms. $W B / \Delta$ is between $0.2-0.4$, while $L W T / L B H$ is concentrated around 0.15 with less abbreviations and hence, high predictability. Subsequently, the mass of the ships hull compared to displacement is somewhat similar in all MPCV, which could let to a reliable prediction tool in steel weight estimations.

Furthermore, since the energy efficiency design index became mandatory for the newbuildings in 2011, the database vessels here are assessed in that manner; EEDI is calculated according to slightly modified IMO formula [17] used here for the preliminary design stage: 


$$
E E D I=\frac{P_{B} \times S C F \times C_{F}}{D W T \times V}
$$

EEDI, for which the distribution can be seen in Figure 8, is obtained at $75 \%$ of the brake engine power $P_{B}$, in $\mathrm{kW}$, necessary to achieve speed $V$, in knots. Specific fuel consumption $S F C$ is input in $\mathrm{g} / \mathrm{kWh}$, conversion factor $C_{F}$ in $\mathrm{gCO}_{2} / \mathrm{t} \cdot$ fuel. In addition, the recent guidelines for attained EEDI calculation are presented in [18]. EEDI results here are compared to the EEDI reference line - EEDI Ref, which contains the criterion established before 2015, for MPCV acquired for over $400 G T$, constructed according to IMO resolution and calculated for this database. Hence, EEDI value for every ship included should be lower than EEDIRef. Furthermore, IMO strengthened the criteria and delivered a $10 \%$ lower reference line that has

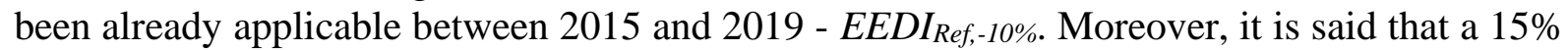
reduction of the reference line is required from 2020 until 2024, labeled as $E E D I_{\text {Ref,-15\%, and }}$ $30 \%$ for the period after the $2025-E E D I_{\text {Ref,-30\%. The }} p d f$ of EEDI registered that a certain number of ships in the database would not be able to meet the present-day, let alone future criteria. More illustrative insight on EEDI criteria can be found in the following section.

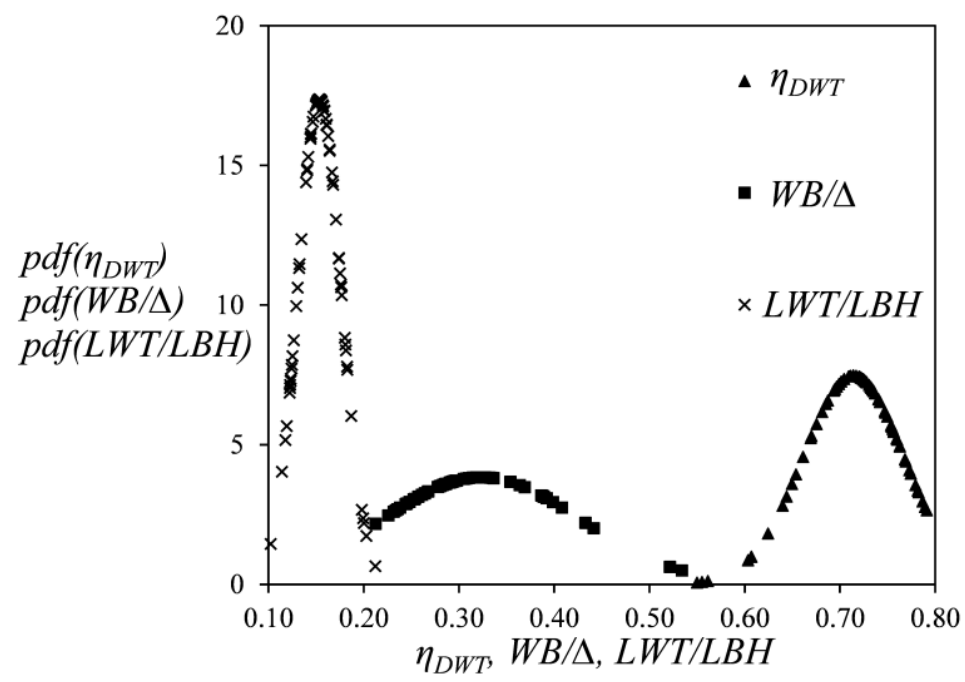

Fig. 7 pdf of $\eta_{D W T}, W B / \Delta$ and $L W T / L B H$

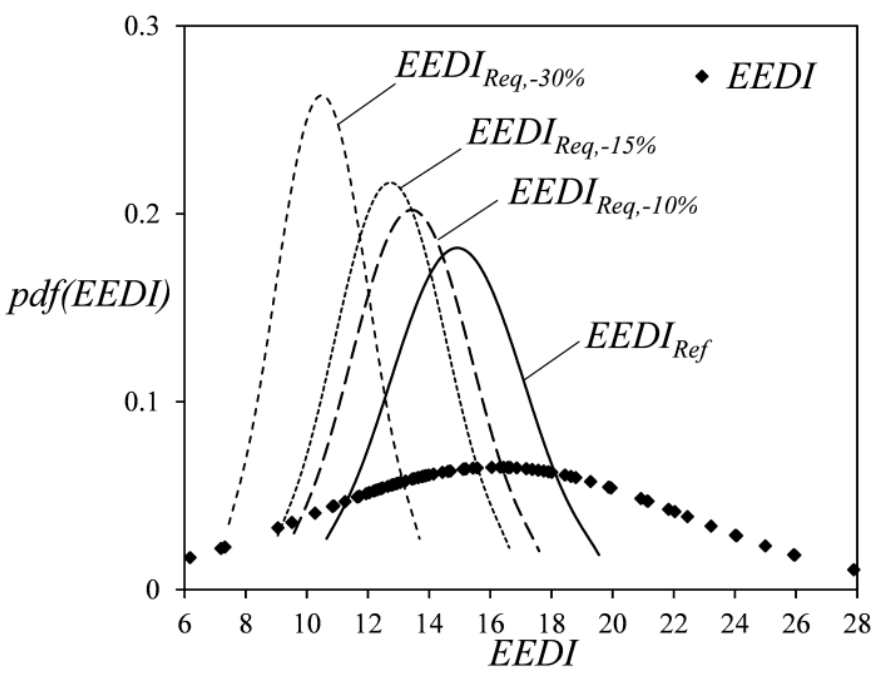

Fig. 8 pdf of EEDI 
Table 1 Statistical parameters of the database

\begin{tabular}{|c|c|c|c|c|c|c|}
\hline & $L_{O A}$ & $B$ & $H$ & $T$ & $C_{B}$ & $F_{n}$ \\
\hline$n$ & 108 & 108 & 101 & 102 & 78 & 78 \\
\hline$E(X)$ & 136.9 & 20.5 & 10.5 & 7.61 & 0.74 & 0.23 \\
\hline$\sigma(X)$ & 32.57 & 5.30 & 3.25 & 1.95 & 0.07 & 0.03 \\
\hline & $P_{B}$ & $P_{M E D A}$ & $\Delta$ & $D W T$ & $L W T$ & $G T$ \\
\hline$n$ & 93 & 47 & 78 & 108 & 78 & 91 \\
\hline$E(X)$ & 6815 & 949 & 17874 & 12943 & 5003 & 10609 \\
\hline$\sigma(X)$ & 4999 & 584 & 12063 & 8885 & 3173 & 7297 \\
\hline & $C C$ & $H F O$ & $D O$ & $W B$ & $\eta_{D W T}$ & $W B / \Delta$ \\
\hline$n$ & 70 & 67 & 77 & 81 & 78 & 70 \\
\hline$E(X)$ & 15984 & 1139 & 184 & 5190 & 0.71 & 0.30 \\
\hline$\sigma(X)$ & 11499 & 835 & 145 & 3331 & 0.053 & 0.10 \\
\hline & $L W T / L B H$ & $E E D I$ & $E E D I_{\text {Ref }}$ & $E E D I_{\text {Req },-10 \%}$ & $E E D I_{\text {Req, }-15 \%}$ & $E E D I_{\text {Req,-30\% }}$ \\
\hline$n$ & 78 & 88 & 100 & 100 & 100 & 100 \\
\hline$E(X)$ & 0.154 & 16.2 & 14.9 & 13.4 & 12.7 & 10.5 \\
\hline$\sigma(X)$ & 0.023 & 6.12 & 2.19 & 1.97 & 1.87 & 1.52 \\
\hline
\end{tabular}

\section{Preliminary design procedure}

Statistical analysis of the database from the previous section is used to obtain diagrams and formulas for MPCV preliminary design. Not all the parameter data can be reliably referred to make predictions because of wider dissipation properties. However, those that can be applied, are presented in diagrams, see Figures 9-15. Trendline formula and their respective coefficients of determination $R^{2}$ is given in equations 3-14.

As the required deadweight is the principal prerequisite for the determination of main ship particulars, so $L_{O A}, B$, and $H$ are to be obtained from Figure 9. The database predicts reliable tendencies as these three parameters offer the least data dissipation: $R^{2}>0.8$. $T$ can be found with similar reliability, see Figure 10. Note that two of outermost points represents ice class MPCV which have increased height compered to the rest of the "standard" vessels.

Since $L, B$, and $H$ are acquired considering $\mathrm{L} \sim 0.95 \mathrm{~L}_{\mathrm{OA}}$, according to the database, one can estimate $G T, L W T$ and $\Delta$ of the particular ship, as in Figure 11, where an additional $D W T$ $L B H$ dependency is also revealed. Water ballast volume for the acquired displacement is predicted with small deviation from the database, as shown in Figure 12. In contrast, $C_{B}$, as a function of $F_{n}$, has rather larger dissipation, $R^{2}=0.58$, although the proposed formula, shown in equation 12, falls in between, as seen in Figure 13, the available literature predictions given by Watson, Ayre, and Schneekluth [2, 3]. Some of these trendlines from [2,3] are based on older ships databases, slenderer forms or ones having low wave resistance forms.

$F_{B}$ can be calculated based on obtained $L_{O A}$, although the data present a larger bandwidth as freeboard is not uniformly distributed along the diversified MPCV fleet, shown in Figure 14. The uppermost marked are ICE class ships and deviate largely from the critical line; the ones marked on the lower part of the diagram are river-sea ships. Note that these three ships are below the reference line issued by ICLL [19] - the minimum value line 
required, obtained without any correction factors, e.g. bow height, minimum reserve buoyancy, etc.

To avoid larger taxes, some ship owners tend to pursue the decrease of the freeboard, and are in danger of delivering stability issues - while positioning the ship near the ICLL line, without proper margin. Different ships are designed for different navigation routes as they have a significant influence on freeboard and respective requirements. Ensuring that all presented ships lie above the ICLL line with a firm margin, more rigid freeboard criteria may be required. This research proposes a trendline, see Figure 14, in which one may estimate a reliable freeboard with an appropriate margin, allowing for safer navigation.

An EEDI trendline and calculation formula is given in Figure 15 and equation 14 to estimate EEDI for the required DWT. IMO reference lines, described in Section 2, are presented to investigate how the contemporary database of MPCV are being referred, regarding the evolution of IMO criterion, starting from before $2015 E E D I_{R e f}$ to reduced criterions for 2015-2019, 2020-2024, and after the 2025, see respectively - EEDI $I_{\text {Req,-10\%, }}$ $E E D I_{\text {Req, }-15 \%}$, and $E E D I_{\text {Req, }-30 \%}$. Note that a large number of present ships cannot pass even the

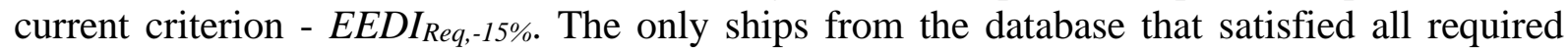
lines are the ones with very low speed: $14-15 \mathrm{kn}$. Therefore, EEDI criteria could be fulfilled if the speed is reduced. However, these ships would be considerably less efficient throughout the year, so for the same amount of cargo, a larger fleet is to be employed. This raises an important concern regarding the extent to which fuel emissions are reduced. The paper is not intended to answer this question. Still, decreasing EEDI by decreasing speed for the single ship - may push the increase of the fleet overall, to accommodate the same annual cargo transport. Nevertheless, it has to be taken into account that simplified EEDI calculation here can be used as an estimation in order to compare the vessels and insight the trends on how the emerging criterions generally affect the overall fleet. More detailed EEDI calculation, as well as reliability-based optimization design can be found in [20].

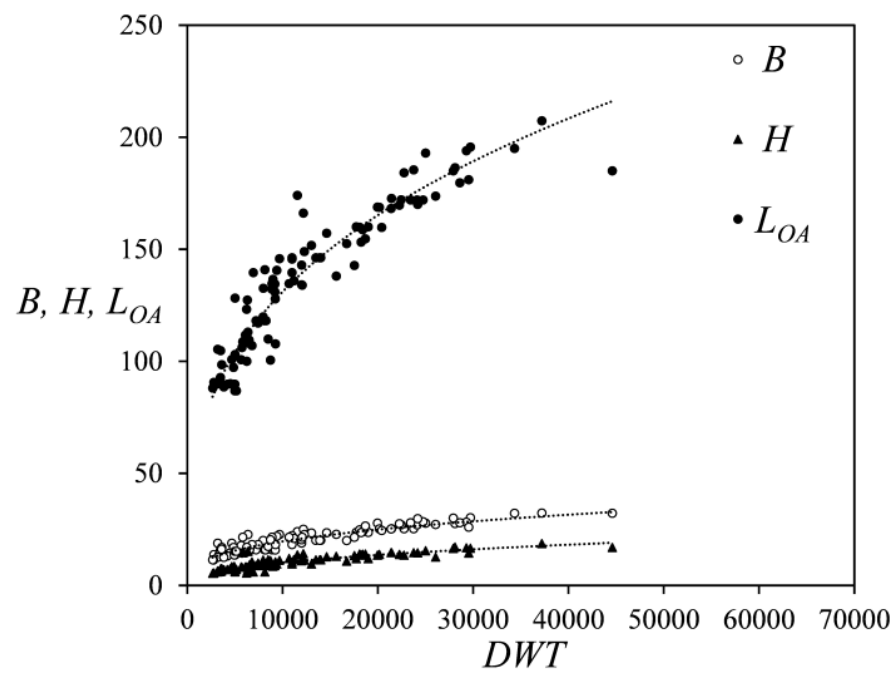

Fig. $9 L_{O A}, B$ and $H-D W T$ 


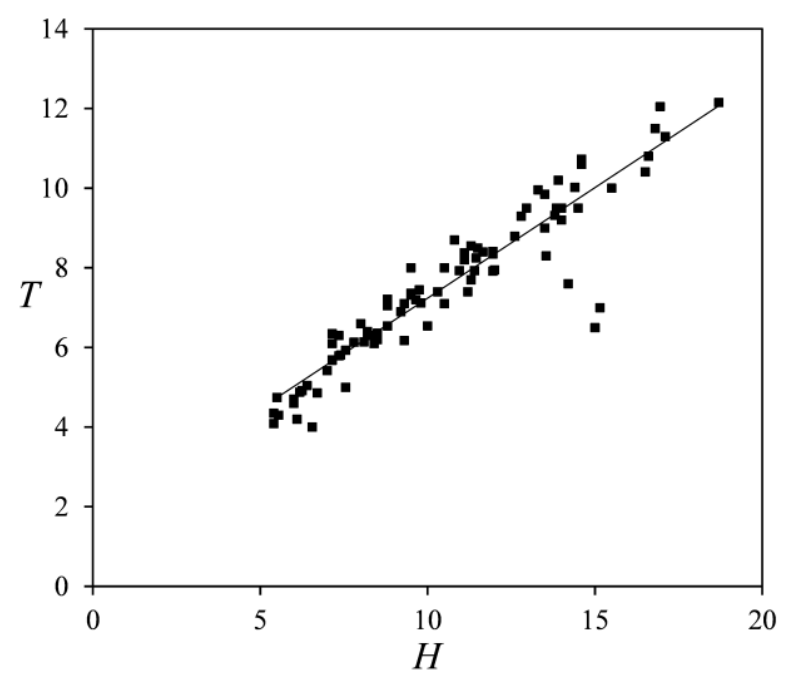

Fig. $10 T-H$

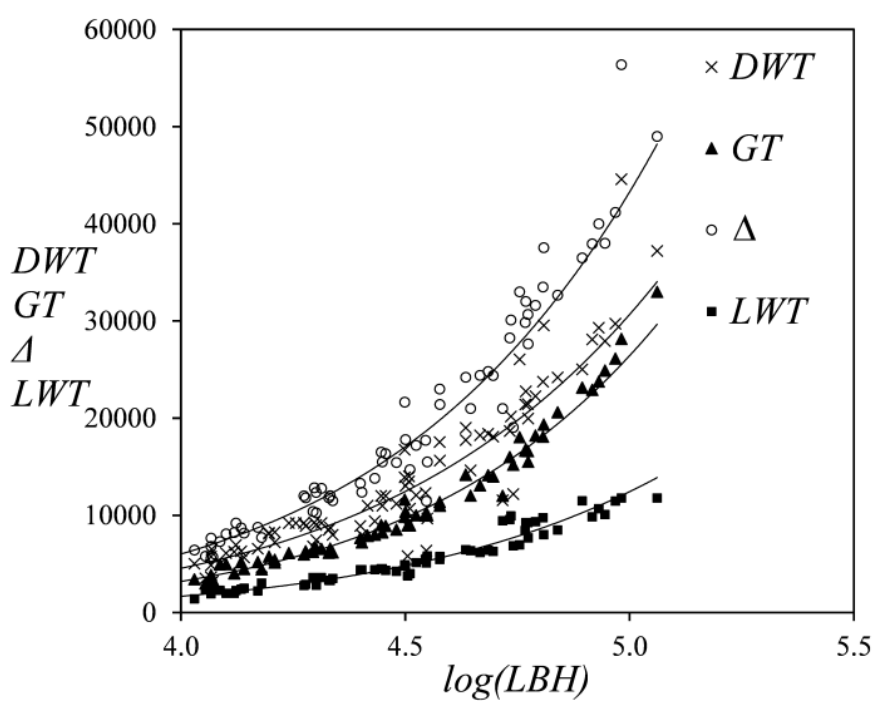

Fig. $11 D W T, G T, \Delta, L W T-L B H$

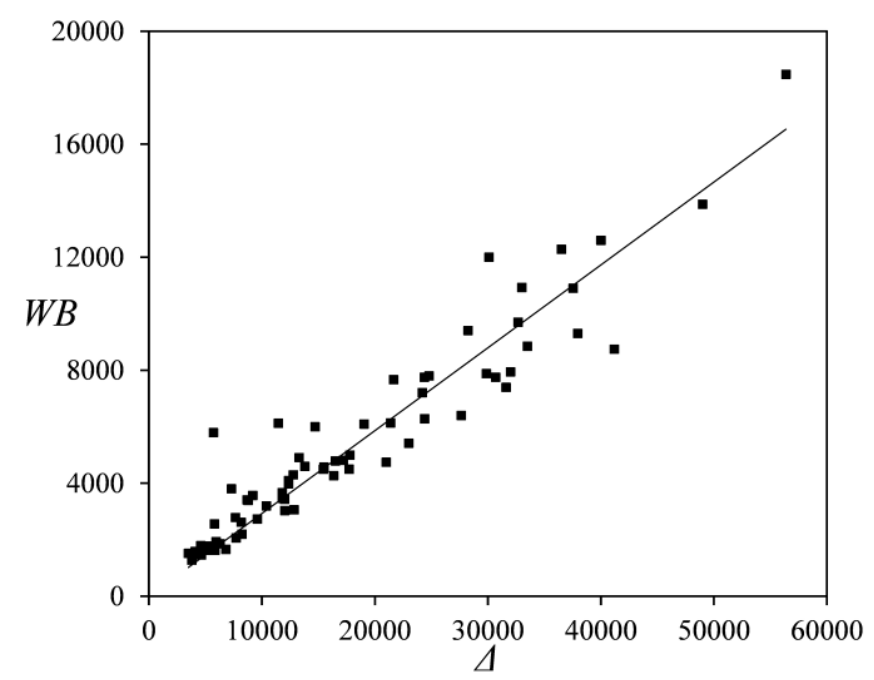

Fig. $12 W B-\Delta$ 

of Seagoing Multi-Purpose Cargo Vessels

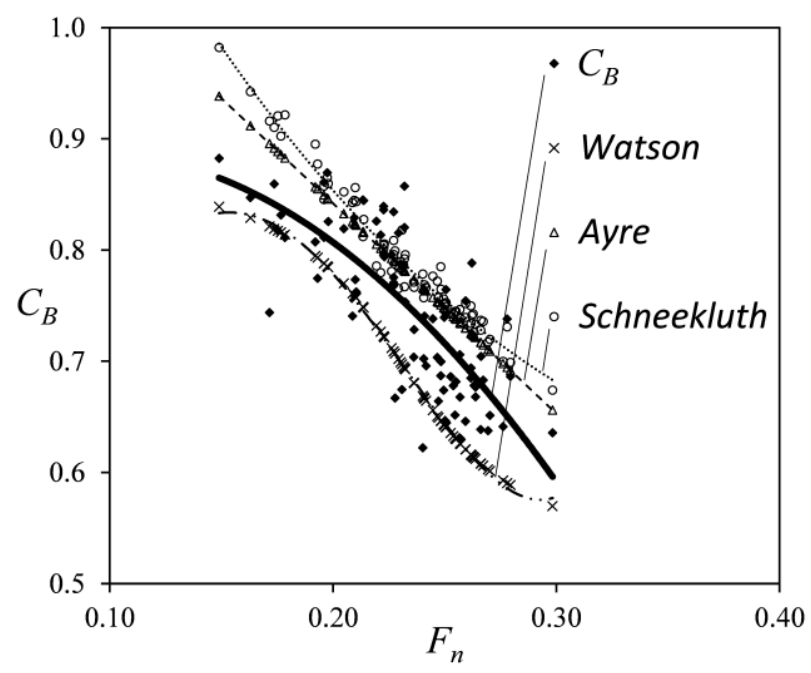

Fig. $13 C_{B^{-}} F_{n}$

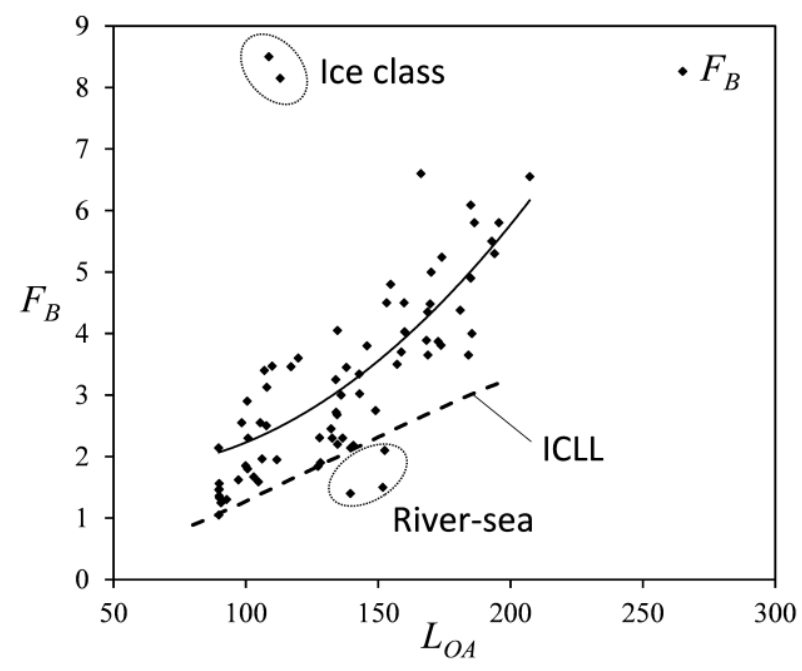

Fig. $14 F_{B}-L_{O A}$

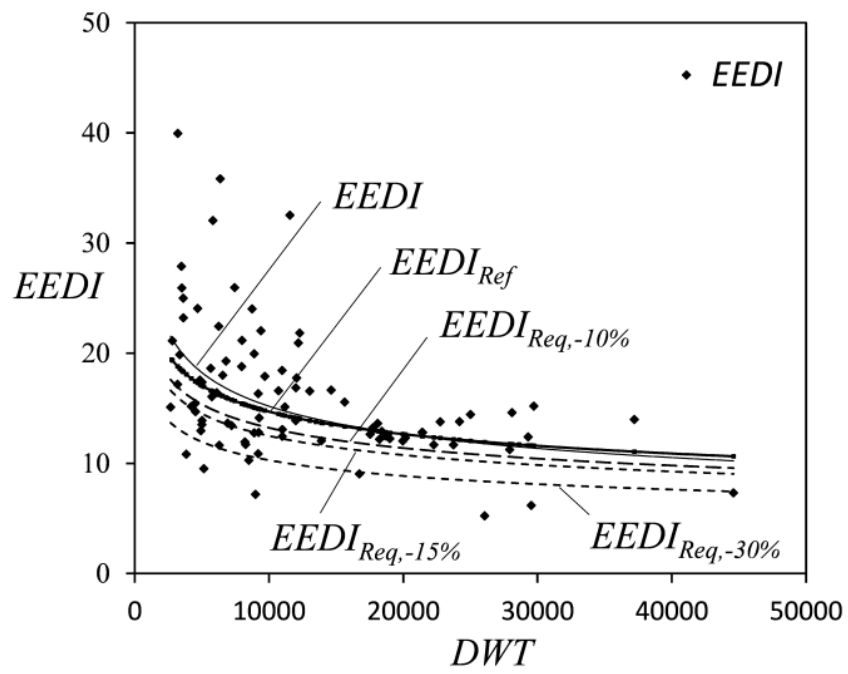

Fig. 15 EEDI-DWT 


$$
\begin{aligned}
& L_{O A}=6.017(D W T)^{0.335}, R^{2}=0.89 \\
& B=0.842(D W T)^{0.342}, R^{2}=0.83 \\
& H=0.217(D W T)^{0.418}, R^{2}=0.80 \\
& \Delta=0.029(L B H)^{8.84}, R^{2}=0.96 \\
& D W T=0.0323(L B H)^{8.551}, R^{2}=0.91 \\
& G T=0.0064(L B H)^{9.46}, R^{2}=0.98 \\
& L W T=0.0065(L B H)^{8.99}, R^{2}=0.97 \\
& T=0.555 H+1.69, R^{2}=0.87 \\
& W B=0.293 \Delta, R^{2}=0.89 \\
& C_{B}=-6.76 F_{n}^{2}+1.224 F_{n}+0.833, R^{2}=0.58 \\
& F_{B}=0.0002 L_{O A}^{2}+0.018 L_{O A}+2.242, R^{2}=0.43 \\
& E E D I=173.38 D W T^{-0.264}, R^{2}=0.26
\end{aligned}
$$

\section{Some notes on freeboard and EEDI}

Since some of the ships are very near the ICLL freeboard reference line and a large number of them could not pass EEDI criteria, a reliability index and probability of exceedance of freeboard and EEDI criteria is assessed. The mathematical procedure is thoroughly explained in [21]. The following should give a closer insight to the present MPCV fleet in terms of its standings along with prescribed criteria.

The reliability index that the specified ship would have $F_{B}$ larger than $F_{B, I C L L}$ is calculated according to Cornell definition [22]:

$$
\beta=\frac{E(M)}{\sigma(M)}=\frac{\frac{1}{E\left(F_{B} / F_{B, I C L L}\right)}-1}{V\left(F_{B}\right)}
$$

$M$ is a margin and is a linear function of independent variables. It stands for the deviation between the ship $F_{B}$ and the benchmark $F_{B, I C L L}: M=F_{B}-F_{B, I C L L}$. All uncertainties are taken to be negligible as $F_{B}$ is calculated straightforwardly. Having in mind that the $p d f\left(F_{B}\right)$ is Gaussian, the probability $P$ that $F_{B}$ will exceed the $F_{B, I C L L}$ and the probability that would not exceed, $1-P$, are obtained as

$$
P(M \geq 0)=\Phi(-\beta) ; 1-P=\Phi(\beta)
$$

The analogous procedure and mathematical assumptions are performed for the calculation reliability index and probabilities of EEDI. Therefore, the reliability index of 
$E E D I$ with respect to the reference lines, $E E D I_{\text {Ref, }}, E E D I_{\text {Req,-10\%, }}, E E D I_{\text {Req,-15\%, }} E E D I_{\text {Req,-30\%, }}$ can be obtained as in equation:

$$
\begin{aligned}
& \beta=\frac{\frac{1}{E\left(E E D I / E E D I_{\mathrm{Re} f}\right)}-1}{V(E E D I)} ; \beta=\frac{\frac{1}{E\left(E E D I / E E D I_{\mathrm{Re} q,-10 \%}\right)}-1}{V(E E D I)} ; \\
& \beta=\frac{\frac{1}{E\left(E E D I / E E D I_{\mathrm{Re} q,-15 \%}\right)}-1}{V(E E D I)} ; \beta=\frac{\frac{1}{E\left(E E D I / E E D I_{\mathrm{Re},-30 \%}\right)}-1}{V(E E D I)}
\end{aligned}
$$

The probability that EEDI would exceed, $P$, any of the reference lines is obtained as in equation 16 . The probability that it would not exceed is $1-P$, which is desirable in this case, as $E E D I$ should be as low as possible, contrary to $F_{B}$. The margin $M$ here is the difference between the $E E D I$ for the specific ship and its reference criteria: $M=E E D I-E E D I_{\text {Ref, }} M=$ $E E D I-E E D I_{\text {Req, }-10 \%,} M=E E D I-E E D I_{\text {Req, }-15 \%}, M=E E D I-E E D I_{\text {Req, }-30 \%}$.

\begin{tabular}{|c|c|c|c|c|c|}
\hline$X$ & $F_{B}$ & \multicolumn{4}{|c|}{ EEDI } \\
\hline Reference & $F_{B, I C L L}$ & $E E D I_{R e f}$ & $E E D I_{\text {Req, }-10 \%}$ & $E E D I_{\text {Req, }-15 \%}$ & $E E D I_{\text {Req, }, 30 \%}$ \\
\hline$\beta$ & -1.61 & -0.28 & -0.51 & -0.62 & -1.02 \\
\hline$-\beta$ & 1.61 & 0.28 & 0.51 & 0.62 & 1.02 \\
\hline$P$ & 0.95 & 0.61 & 0.70 & 0.73 & 0.85 \\
\hline $1-P$ & 0.05 & 0.39 & 0.30 & 0.27 & 0.15 \\
\hline
\end{tabular}

Table 2 Reliability index and probability of exceedance/non exceedance

The probability of $F_{B}$ exceedance $P=0.95$ refers that the probability that $F_{B}$ of each ship of the database would pass the ICLL criterion, is $95 \%$, which can be assessed as quite large, see table 2. However, notice that three river-sea vessels out layered the line due to their low freeboard considering their specific navigation area and large number of ships are near reference. These particular ships, although seemingly insignificant, should not been neglected as their frequent cargo-draught change allows for their use in more coastal areas. The proposed trendline has a goal of delivering such freeboard that can ensure safe margins with regard to required ICLL criteria.

Furthermore, the probability that the current EEDI of the ships passes the criteria used before 2015 is 0.39 , see $1-P$ in table 2, since EEDI should be as low as possible, implicating low emissions. The 1-P for the period 2015-2019 is even smaller - 0.30, as just $30 \%$ of the database fleet satisfied the second criteria. The current reference line for 2020-2024, is further reduced, so the probability that these ships would satisfy the criterion drops to $27 \%$. Future trends are even worse if these ships were still to navigate. As discussed in the previous section, decreasing speed should not be the only solution for shipowners, as this may produce larger fleet that would lead to greater emissions overall.

A reliability analysis illustrated the range of present fleet within the scope of two influential design criteria that should be kept in mind even in the preliminary phase: freeboard and energy efficiency design index. These issues are to be assessed seriously since the main ship parameters governing $F_{B}$ and $E E D I$ are very difficult to change in subsequent stages of the design spiral. 


\section{Conclusion}

The aim of this paper is to build a reliable database and respective trendlines that can be used as a practical tool in a preliminary design stage, in case of multi-purpose cargo vessels built over the past 25 years. This paper was prompted by the lack of systematic literature data on modern medium sized MPCV, as this niche market has been increasing over the last few years. Based on the required deadweight, one can estimate the length, breadth, height, draught of the vessel. Furthermore, a block coefficient and Froude number, and therefore, speed - can be calculated; as well as displacement, lightweight, deadweight mass, gross tonnage and water ballast capacity.

Furthermore, freeboard and energy efficiency of current fleet are estimated and compared to the reference lines issued by IMO. Moreover, a reliability analysis is performed to emphasize that the designer should consider the freeboard and especially energy efficiency design indices carefully. Freeboard could be critical for emerging fleet of coastal, riversea/sea-river multi-purpose cargo vessels and might need to be addressed in a criterion itself. This paper intended to present a freeboard margin that should be considered when designing a new MPCV, in comparison to the required minimum. However, for that purpose, a larger and more diversified databases of the specific MPCV should be assessed.

Additionally, in the case of EEDI considerations, contemporary fleet could not satisfy old, let alone current or future criteria. Speed reduction is an obvious solution that could led to a fleet increase for the same cargo carried, and in that case a single ship emission issue would become problematic on a more global scale.

Although preliminary design stage is looking as a single step in many of them; it is probably the most important one, as the estimation of main ship parameters could direct to hardly repairable design on the end of the process.

\section{ACKNOWEDGEMENT}

This work was supported by Ministry of Education, Science and Technological Development (Project no. TR 35009) of Serbia.

\section{REFERENCES}

[1] Papanikolaou A., Ship Design - Methodologies of Preliminary Design, Springer, 2014. https://doi.org/10.1007/978-94-017-8751-2

[2] Bertram V. and Schneekluth H., Ship Design for Efficiency and Economy, Second Edition, ButterworthHeinemann, 1998.

[3] Watson D.G.M., Practical Ship Design, Vol. I, Elseiver, 1998.

[4] Grubišić R., Kučan E., Radelja N, Rimanić R., Vlak R., Sablić D., Maletić M., Grubisa R. and Dragovic M, An 18680 dwt Multipurpose/Heavy Lift Cargo Vessel, Part I, Brodogradnja, Vol. 59, No. 4, pp. 348356, 2008.

[5] Grubišić R., Grubiša R., Rimanić R., Vlak R., Kučan E. and Štokic M., An 18680 dwt Multipurpose/Heavy Lift Cargo Vessel, Part II, Brodogradnja, 2009, Vol. 60, No. 2, pp. 157-162, 2009.

[6] Moustafa M. M., Optimization Procedure for Preliminary Design Stage of Cairo-Damietta Self-Propelled Grain Bulk Ships, Brodogradnja, Vol. 66, No. 4, 2015.

[7] Lamb T. (editor), Ship Design and Construction, SNAME, Vol. I, 2003.

[8] Charchalis A., Determination of Main Dimensions and Estimation of Propulsion Power of a Ship, Journal of KONES Powertrain and Transport, Vol. 21, No. 2, pp. 39-44, 2014. https://doi.org/10.5604/12314005.1133863 
[9] Abramowski T., Cepowski T. and Zvolensky P., Determination of Regression Formulas for Key Design Characteristics of Container Ships at Preliminary Design Stage, New Trends in Production Engineering, Vol. 1, No. 1, pp. 247-257, 2018. https://doi.org/10.2478/ntpe-2018-0031

[10] Kristensen H.O., Statistical Analysis and Determination of Regression Formulas for Main Dimensions of Container Ships based on IHS Fairplay Data, Report no. 3, Technical University of Denmark, 2013.

[11] Evans J., Basic Design Concepts. Naval Engineers Journal, pp. 671-678, 1959. https://doi.org/10.1111/j.1559-3584.1959.tb01836.x

[12] Van Bruinessen T., Hopman J. and Smulders F., Towards a Different View on Ship Design: The Development of Ships Observed Through a Social - Technological Perspective, 32nd International Conference on Ocean, Offshore and Arctic Engineering ASME 2013, 2013. https://doi.org/10.1115/OMAE2013-11585

[13] Equasis, The World Merchant Fleet in 2017- Statistics from Equasis, 2017.

[14] Kalajdžić M. and Momčilović N., On Preliminary Design of Multi-Purpose Cargo Vessels, IMSC2019 conference, Budva, Montenegro, 2019.

[15] RINA, Significant Ships, 1995-2019.

[16] Damen, Multi purpose Vessel (https://products.damen.com/en/clusters/multi-purpose-vessel-shipping).

[17] IMO, Resolution MEPC.203 (62), London, 2011.

[18] IMO, Resolution MEPC.308(73), London, 2018.

[19] IMO, International Convention on Load Lines, 1998.

[20] Hang H.Y., Xiao L. and Yang M.X., Hull Lines Reliability-Based Optimization Design for Minimum EEDI, Brodogradnja, Vol. 69, No. 2, 2018. https://doi.org/10.21278/brod69202

[21] Mansour A. and Liu D., Strength of Ships and Ocean Structures, SNAME, 2008.

[22] Cornell C.A., A Probability-Based Structural Code, Journal of the ACI, Vol. 66, No. 12, pp. 974-985, 1969. https://doi.org/10.14359/7446

Submitted: $\quad$ 09.03.2020. Milan Kalajdžić, mdkalajdzic@mas.bg.ac.rs

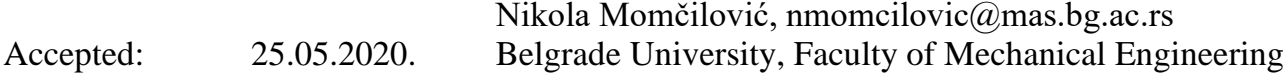
Kraljice Marije 16, 11120 Belgrade 35 